\title{
Ad-endostatin treatment combined with low-dose irradiation in a murine lung cancer model
}

\author{
XIAO-PENG LI ${ }^{1 *}$, HAI-LONG ZHANG ${ }^{1 *}$, HUI-JUAN WANG ${ }^{1 *}$, YONG-XIA LI $^{1}$, MENG LI $^{1}$, \\ LIAN LU $^{1}$, YANG WAN ${ }^{1}$, BAI-LING ZHOU ${ }^{1}$, YAN LIU ${ }^{1}$, YING PAN ${ }^{1}$, XIAO-ZHE WU ${ }^{1}$, \\ YING-ZI FAN $^{1}$, CHAO-HENG YU ${ }^{1}$, YU-QUAN WEI ${ }^{1}$ and HUA-SHAN SHI ${ }^{1,2}$
}

\author{
${ }^{1}$ State Key Laboratory of Biotherapy and Cancer Center, West China Hospital, West China Clinical Medical School, \\ Sichuan University; ${ }^{2}$ State Key Laboratory of Biotherapy and Department of Head and Neck Oncology, \\ West China Hospital, West China Medical School, Sichuan University, Chengdu, Sichuan 610041, P.R. China
}

Received January 27, 2014; Accepted March 7, 2014

DOI: $10.3892 /$ or.2014.3253

\begin{abstract}
Radiation therapy is a conventional strategy for treating advanced lung cancer yet is accompanied by serious side-effects. Its combination with other strategies, such as antiangiogenesis and gene therapy, has shown excellent prospects. As one of the potent endogenous vascular inhibitors, endostatin has been widely used in the antiangiogenic gene therapy of tumors. In the present study, LL/2 cells were infected with a recombinant adenovirus encoding endostatin (Ad-endostatin) to express endostatin. The results showed that LL/2 cells infected with the Ad-endostatin efficiently and longlastingly expressed endostatin. In order to further explore the role of Ad-endostatin combined with irradiation in the treatment of cancer, a murine lung cancer model was established and treated with Ad-endostatin combined with low-dose irradiation. The results showed that the combination treatment markedly inhibited tumor growth and metastasis, and prolonged the survival time of the tumor-bearing mice. Furthermore, this significant antitumor activity was associated with lower levels of microvessel density and anoxia factors in the Ad-Endo combined with irradiation group, and with an increased apoptotic index of tumor cells. In addition, no serious side-effects were noted in the combination group. Based on our findings, Ad-endostatin combined with low-dose irradiation may be a rational alternative treatment for lung cancer and other solid tumors.
\end{abstract}

Correspondence to: Professor Yu-Quan Wei or Professor Hua-Shan Shi, State Key Laboratory of Biotherapy and Cancer Center, West China Hospital, West China Clinical Medical School, Sichuan University, 4 Keyuan Road, Chengdu, Sichuan 610041, P.R. China

E-mail:272546518@qq.com

E-mail: 41083995@qq.com

${ }^{*}$ Contributed equally

Key words: radiation therapy, adenovirus, endostatin, combination therapy, lung cancer

\section{Introduction}

Lung cancer is a malignancy with high incidence and mortality rates. Woldwide, $13 \%$ of patients with tumors are diagnosed with lung cancer, and the mortality due to lung cancer accounts for $18 \%$ of all cancer-related deaths $(1,2)$. Conventional strategies for treating advanced lung cancer include surgery, chemotherapy and radiotherapy. However, most patients do not have the opportunity to undergo surgical resection when they are diagnosed with lung cancer, and the common therapeutic strategy is radiation therapy. While radiotherapy has shown commendable antitumor activity, serious side-effects always accompany treatment. These include radiation-induced lung injury and radioactive tracheal injury, resulting in the discontinuation of antitumor treatment limiting its application (3-6). Clearly, new therapeutic strategies are imminently needed to optimize available regimens.

Angiogenesis plays an important role in a large variety of physiological and pathological conditions, such as embryonic development, wound healing and diabetic retinopathy $(7,8)$. Evidence indicates that most malignant solid tumor growth and metastases are partly dependent on angiogenesis (9-11). Accordingly, antiangiogenesis is an effective strategy of antitumor treatment by preventing new vessel formation in tumors and destroying the abnormal microvascular system which provides oxygen and nutrients to the tumor tissue.

Endostatin, originally purified from the culture supernatant of murine hemangioendothelioma (EOMA) cells, is a type of endogenous angiogenesis inhibitor, which locates in the basement membrane of blood vessels (12). Amino acid sequence analysis shows that endostatin is a $20-\mathrm{kDa}$ C-terminal fragment of collagen XVIII which belongs to the multiplexin family $(13,14)$. Extensive basic and preclinical research reveals that endostatin not only inhibits the proliferation and migration of endothelial cells, but also induces the apoptosis of vascular endothelial cells, thereby suppressing angiogenesis (15-17). Further studies have demonstrated that endostatin also inhibits the growth and metastasis of many types of solid tumors. Therefore, endostatin targeting angiogenesis has been increasingly used in preclinical and clinical research $(12,18,19)$. Most therapeutic strategies of endostatin 
have utilized the purified protein $(20,21)$. However, as a protein drug, endostatin has a short half-life in vivo, and thus needs continuous administration or increased concentrations to maintain effective serum levels. Moreover, the process of protein purification may denature endostatin, which reduces the resultant yield rates. These problems seriously hamper the clinical application of endostatin $(2,22)$. One possible strategy to overcome these issues is the utilization of gene therapy, and adenoviral vectors are widely utilized for gene therapy and show no disadvantages of purified protein injection $(2,23-25)$.

Although tumor treatment with endostatin has shown various effects, the inhibitory effect of endostatin is limited since numerous factors are secreted by endothelial cells to promote angiogenesis, while endostatin inhibits just one of these angiogenic factors (3). In addition, the endothelium can acquire resistance to angiogenesis inhibitors through epigenetic changes as recently demonstrated (26). Thus, the combination of endostatin with other treatment strategies (such as radiotherapy) may improve the antitumor effect of endostatin.

In the present study, we established an LL/2 cell subcutaneous tumor model and a lung metastasis model, and evaluated the efficacy of the combined treatment of recombinant endostatin adenovirus (Ad-Endo) with low-dose irradiation. The present study revealed that this strategy enhances the antitumor effect without serious side-effects, and provides a rational alternative treatment for lung cancer and other solid tumors.

\section{Materials and methods}

Cell culture and Ad-Endo preparation. Lewis lung carcinoma (LL/2) and human embryonic kidney (HEK293) cell lines were obtained from the American Type Culture Collection (ATCC; Manassas, VA, USA). They were cultured in Dulbecco's modified Eagle's medium (DMEM) which included 10\% fetal bovine serum (FBS) plus amikacin and streptomycin routinely. The viral particles which were amplified in HEK293 cells were purified by $\mathrm{CsCl}$ gradient ultracentrifugation and measured by absorption (at A260). The viral titer was quantified with the standard 50\% tissue culture infectious dose (TCID50) assay.

Determination of the multiplicity of infection (MOI). LL/2 cells were infected with Ad-GFP at multiple MOIs [5, 10, 50, 100 and 200, the plaque-forming units (pfu)/cells in $1.0 \mathrm{ml}$ complete medium) or involved no infection. After culturing at $37^{\circ} \mathrm{C}$ for $48 \mathrm{~h}$, the cells containing GFP were observed by fluorescence microscopy and then subjected to flow cytometry and analyzed using CellQuest software.

Detection of endostatin expression by western blot analysis. LL/2 cells were infected with Ad-Endo and Ad-null at an MOI of 100 or not infected. Cells were conditioned at $37^{\circ} \mathrm{C}$ for $48 \mathrm{~h}$, and the supernatant was collected and concentrated by ultrafiltration (Millipore, Darmstadt, Germany). The retentate was mixed with 5X SDS sample buffer and separated on a 12\% SDS-PAGE gel and then transferred onto a polyvinylidene difluoride (PVDF) membrane. After the membrane was blocked by TBST containing 5\% defatted milk for $2 \mathrm{~h}$, it was probed with goat anti-human endostatin polyclonal antibody $\left(1: 1,000\right.$; R\&D Systems) overnight at $4^{\circ} \mathrm{C}$, followed by horseradish peroxidase-conjugated anti-goat immunoglobulin (1:5,000; ZSGB-BIO, Beijing, China) for $2 \mathrm{~h}$ at $37^{\circ} \mathrm{C}$. Three washes (15 min per wash) were carried out after each incubation step. The protein bands were detected by an enhanced chemiluminescence detection system (Pierce, Rockford, IL, USA).

Determination of transgene expression by enzyme-linked immunosorbent assay. Human endostatin expression in the supernatant of cultured cells or in mouse serum was detected with the human endostatin ELISA kit (Sigma-Aldrich, St. Louis, MO, USA) according to the manufacturer's instructions. For the supernatant of cells infected with viral particles, the supernatant was collected for testing after 24,48 and $72 \mathrm{~h}$. For the mouse serum, on days 1, 7, 14 and 21 after tail vein administration of viral particles $\left(1 \times 10^{9} \mathrm{pfu}\right)$ in the LL/2 cell subcutaneous tumor model, the blood samples were obtained and centrifuged for $10 \mathrm{~min}$ at $3,000 \mathrm{rpm}$, and then the serum was collected for testing.

The LL/2 subcutaneous tumor model and the in vivo antitumor effect of Ad-Endo combined with radiotherapy. To evaluate the antitumor effect of Ad-Endo combined with radiotherapy in the established animal model, C57BL/6 female mice (6-8 weeks of age) were injected s.c. with $3 \times 10^{6} \mathrm{LL} / 2$ cells in $100 \mu 1$ phosphate-buffered saline (PBS) in the right flank. Seven days later, the tumors were palpable on each mouse, and the mice were then randomly divided into 6 groups (10 mice/group). i) Ad-Endo group received an i.v. injection of $1 \times 10^{8} \mathrm{pfu} / 100 \mu \mathrm{l}$ recombinant adenovirus. ii) RT group received irradiation performed with the radiation treatment system Elekta Compact $^{\text {TM }}$ (Elekta AB, Stockholm, Sweden) at a dose of $2 \mathrm{~Gy}$ and locally operated on the tumors by shielding the rest of the body. iii) Ad-Endo plus RT group received Ad-Endo administration i.v. and 2 Gy dose of irradiation confined to the tumors. iv) Ad-null group received an i.v. injection of $1 \times 10^{8} \mathrm{pfu} / 100 \mu \mathrm{l}$ control adenovirus. v) Ad-null plus RT group received Ad-null administration i.v. and 2 Gy dose of irradiation confined to tumors. vi) NS group received an i.v. administration of $100 \mu \mathrm{l}$ of PBS at the same schedule as the other groups. When the groups were completed, treatment was initiated at this time (day 0 ). The viral particles were administered once every three days from day 0 , and the irradiation was delivered each day from day 2 to 6 and day 9 to 13 .

Tumor growth was monitored by measuring tumor width (W) and length (L) every three days. The tumor volume (V) was calculated using the fomula: $\mathrm{V}=0.52 \times \mathrm{L} \mathrm{x} \mathrm{W}^{2}$. The body weight of mice was monitored every three days until day 22 after treatment. On day 22 , the mice were sacrificed, and the major organs were obtained for histologic analysis. All experiments involving mice were guided by the Animal Care and Use Committee of our institute.

To assay the survival time, 60 mice were challenged with LL/2 cells as above. When the tumors were palpable, the mice were grouped and treated as above. The time of death of each mouse was recorded.

Assessment of tumor vascularization and anoxia factors. To analyze the mechanism of the enhanced antitumor effect of Ad-Endo combined with irradiation, the tumor microvessel 
density (MVD) and the level of anoxia factors was detected by immunohistochemistry of CD31 and HIF-1 $\alpha$.

The quantification of MVD was carried out as previously described (27). Briefly, when the mice were sacrificed, the tumors were obtained and fixed with formalin. Then the paraffin-embedded sections of the tumors were probed with a monoclonal rat anti-mouse CD31 antibody (1:400; Santa Cruz Biotechnology, Santa Cruz, CA, USA), followed by a biotinylated polyclonal rabbit anti-rat IgG antibody $(1: 200$; Vector Laboratories, Burlingame, CA, USA). The positive reaction was detected using the DAB substrate kit (Vector Laboratories), and the sections were counterstained with hematoxylin. Finally, the microvessels were calculated with an Olympus microscope at a x200 magnification.

The levels of anoxia factors in the tumors were detected as followed. First, the tumors were obtained and fixed with formalin. Then the paraffin-embedded sections were probed with a monoclonal rabbit anti-mouse HIF-1 $\alpha$ antibody (1:200, Abcam, Cambridge, MA, USA), followed by a biotinylated goat anti-rabbit IgG antibody (1:200; Vector Laboratories). A positive reaction was detected using the DAB substrate kit, and sections were counterstained with hematoxylin. Finally, the sections were mounted with glass coverslips, and images were captured with an Olympus microscope at a 200 magnification.

Detection of apoptosis. The apoptotic cells in the tumor tissues were detected using the terminal deoxynucleotidyl transferasemediated nick end labeling assay (TUNEL) (In Situ Cell Death Detection kit; Roche, Basel, Switzerland), according to the manufacturer's guidelines. Images were obtained by an Olympus fluorescence microscope at a x200 magnification. The apoptotic cells were counted in 5 high power fields in each slide. The percentage of apoptotic cells among the total number of tumor cells was calculated as the apoptotic index.

Alginate encapsulation assay. The alginate encapsulation assay was performed as previously described (28). Briefly, LL/C cells were resuspended with $1.5 \%$ sodium alginate $(\mathrm{m} / \mathrm{v}$; Sigma-Aldrich), and then the suspension was added into a oscillation pool containing $250 \mathrm{mM} \mathrm{CaCl}_{2}$ to form alginate beads. Each bead contained $1 \times 10^{5} \mathrm{LL} / 2$ cells, and each mouse was implanted s.c. with four beads on the back. The mice were treated with Ad-Endo or/and irradiation as above, and with Ad-null or saline as control. On day 15, the mice were administered i.v. $100 \mu \mathrm{l}$ of FITC-dextran solution $(100 \mathrm{mg} /$ $\mathrm{kg}$; Sigma Chemicals) and sacrificed $20 \mathrm{~min}$ later. Images of the alginate beads were captured using a SPOT Flex camera. The beads were then removed and were transferred into tubes containing $2 \mathrm{ml}$ of physiological saline. After being pounded, the beads were rested for $1 \mathrm{~h}$ and centrifuged for $5 \mathrm{~min}$ at $1,000 \mathrm{x}$. The supernatant was collected and the fluorescence was determined to assess blood vessel formation.

The LL/2 cell pulmonary metastasis tumor model and the in vivo antitumor effect of Ad-Endo combined with radiotherapy. The 6- to 8-week-old female C57BL/6 mice were injected with $3 \times 10^{5} \mathrm{LL} / 2$ cells in $100 \mu \mathrm{l}$ PBS by tail vein. Three days later, the mice were randomly divided into 6 groups identical to those described above. On the same day, the mice were treated with viral particles or/and irradiation as above.
On day 25 , the mice were sacrificed, and the lungs were obtained. The weight and metastatic nodules of the lungs were determined. The lungs were then fixed with formalin for histological analysis.

Observation of toxicity. Drug toxicity indices, such as weight loss, ruffled fur, diarrhea, anorexia, skin ulcerations and behavior change, were closely observed during the entire couse of treatment. In addition, the major organs (heart, liver, spleen, lung and kindey) were fixed in $4 \%$ paraformaldehyde, and embedded in paraffin to obtain sections. The sections were stained with hematoxylin and eosin $(\mathrm{H} \& \mathrm{E})$ and observed using the double-blind method.

Statistical analysis. SPSS 17.0 was used for statistical analysis. For comparison of individual time-points, differences between groups were tested by performing analysis of variance (ANOVA) and the unpaired Student's t-test. A diference was regarded as significant at $\mathrm{P}<0.05$.

\section{Results}

Determination of the MOI. LL/2 cells were infected with multiple MOIs (5, 10, 50, 100 and 200) of Ad-GFP. Forty-eight hours later, the level of GFP in cells was detected with fluorescence microscopy. As shown in Fig. 1A, with the increase in MOI, the proportion of LL/2 cells expressing GFP was increased from 3.1 to $98 \%$. Although there were no differences in proportion between MOI 100 and MOI 200, the cells which were infected with MOI 200 were seriously damaged. Similar results were obtained through flow cytometry (Fig. 1B). These results indicated that Ad-GFP could effectively infect LL/2 cells, in a concentration-dependent manner. Based on this, we selected MOI 100 to continue the following studies.

Expression of recombinant human endostatin in vitro and in vivo. LL/2 cells were infected with Ad-Endo or Ad-null at MOI 100. Forty-eight hours later, the culture supernatants were collected and concentrated to detect the expression of endostatin using western blot analysis. As shown in Fig. 2A, a distinct band $\sim 20 \mathrm{kDa}$, similar to the volume of endostatin, was visualized in the supernatants of the Ad-Endo-infected cells, but not in that of the control cells.

To further analyze the level and duration of endostatin, we detected the level of endostatin in the culture supernatants of LL/2 cells, which were separately infected with Ad-Endo or Ad-null for 24, 48 and $72 \mathrm{~h}$ using ELISA. The results showed that after $24 \mathrm{~h}$, in the LL/2 cells infected with Ad-Endo, endostatin was detected in the supernatants and the level reached $420 \pm 30 \mathrm{ng} / \mathrm{ml}$ (Fig. 2B). The level peaked $(560 \pm 40 \mathrm{ng} / \mathrm{ml})$ after $48 \mathrm{~h}$ and decreased slightly after $72 \mathrm{~h}$ $(490 \pm 25 \mathrm{ng} / \mathrm{ml})$. However, we could not detect the expression of endostatin in the cells which were infected with Ad-null or in cells not infected. Similar results also were detected in vivo (Fig. 2C). These observations indicated that LL/2 cells infected with Ad-Endo stably and persistently expressed secretory endostatin.

Combination treatment significantly reduces tumor growth and prolonges the life-span in the LL/2 cell subcutaneous tumor 
A
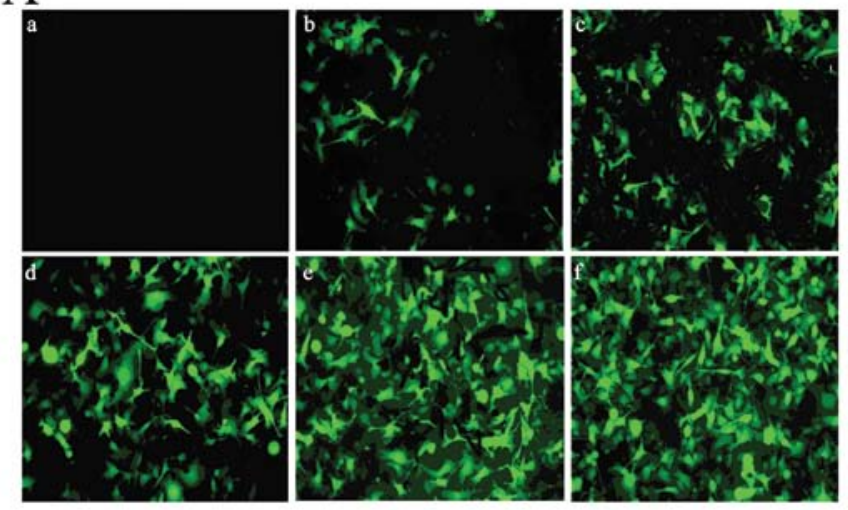

$\mathrm{B}$
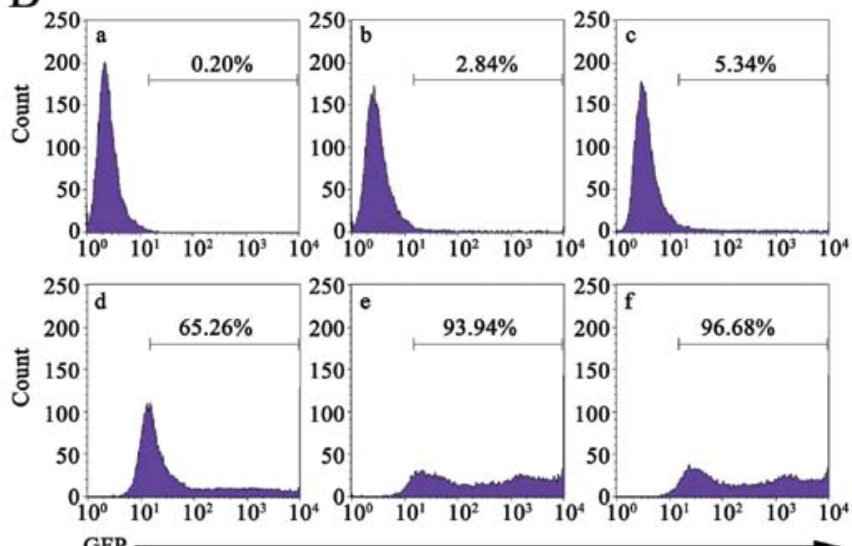

GFP

Figure 1. Analysis of LL/2 cells infected with multiple MOIs of Ad-Endo LL/2 cells were (A) observed by fluorescence microscopy and (B) analyzed using flow cytometry $72 \mathrm{~h}$ after infection with Ad-Endo. (a) LL/2 cells not infected with Ad-Endo, (b) LL/2 cells infected with Ad-Endo at MOI=5, (c) $\mathrm{LL} / 2$ cells infected with Ad-Endo at MOI=10, (d) LL/2 cells infected with Ad-Endo at MOI=50, (e) LL/2 cells infected with Ad-Endo at $\mathrm{MOI}=100$, (f) $\mathrm{L} / 2$ cells infected with Ad-Endo at $\mathrm{MOI}=200$.

model. To assay the antitumor effect of Ad-Endo combined with low-dose irradiation, the LL/2 cell subcutaneous tumor model was established, and the mice were randomly divided into 6 groups after 7 days. The mice were then treated as described in Materials and methods, and the tumor growth was monitored every 3 days. As shown in Fig. 3A, the tumors in the Ad-Endo + RT group were much smaller than tumors in the other groups. Similar to the results in Fig. 3A, the tumor growth rate in the RT group and Ad-Endo group was relatively slower than that in the NS and Ad-null groups $(\mathrm{P}<0.05)$. Compared with the RT, Ad-Endo and Ad-null + RT groups, the combination Ad-Endo + RT group exhibited a more marked inhibition of tumor growth, and the difference was noted from day 16 after treatment. Dramatically, from day 4 after treatment, the tumor growth rate in the combination group showed significant differences when compared with the NS and Ad-null groups. The inhibitory effect was determined by the weight reduction. At the end of the present study, the tumors were removed and measured (data not shown). Compared with the NS group, the tumor weight in the Ad-Endo + RT group was reduced $79 \%$, which was higher than that in the other groups (58.4\% in RT group, $52 \%$ in Ad-Endo group, and $61.7 \%$ in Ad-null + RT group). Furthermore, the combination
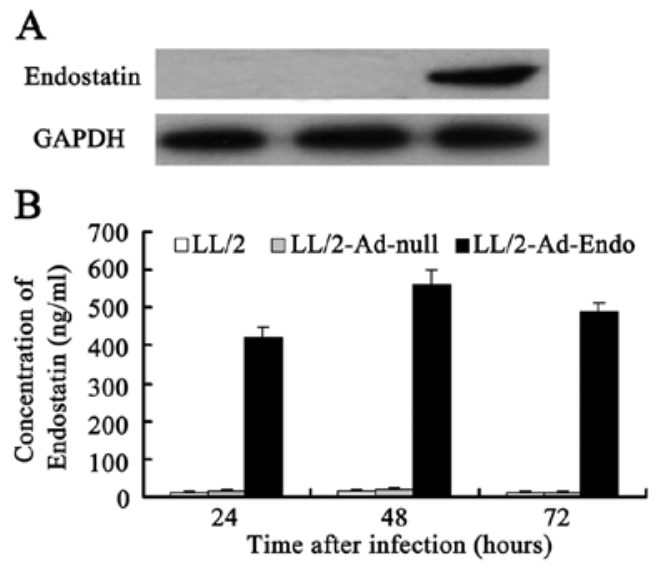

C

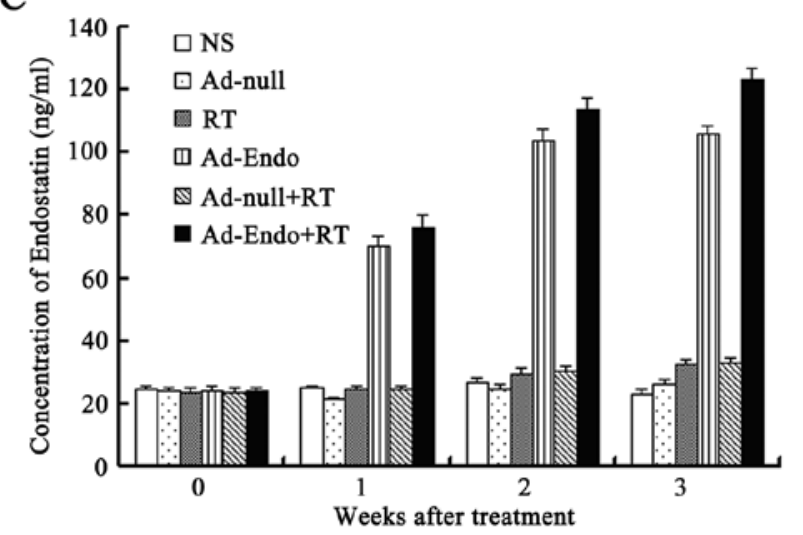

Figure 2. Analysis of the expression and concentration of endostatin in vitro and in vivo. (A) Western blotting. Lane a, the culture supernatant of LL/2 cells not infected with the adenovirus; lane $b$, the culture supernatant of LL/2 cells infected with Ad-null; lane c, the culture supernatant of LL/2 cells infected with Ad-Endo. Moreover, GAPDH is the nal control of this experiment. (B) The ELISA assay. The LL/2 cells were infected with Ad-null or Ad-Endo, and the culture supernatant of LL/2 cells was selected after 24, 48 and $72 \mathrm{~h}$. The concentration of endostatin in the supernatant was analyzed by ELISA, and the culture supernatant of LL/2 cells which had not been infected with the adenovirus was the control. (C) Analysis of the concentration of endostatin in mouse serum. The mouse serum was obtained before treatment and every week after treatment, and the concentration of endostatin in the serum was analyzed by ELISA. Ad-Endo caused a constant high level of endostatin expression, and RT increased the level.

group significantly showed a longer life-span than that of the other groups (Fig. 3C). These results revealed that Ad-Endo + RT improved the antitumor effect of radiotherapy and that of Ad-Endo alone, which reflects the synergism between radiotherapy and Ad-Endo.

Inhibition of tumor-induced angiogenesis and increase in apoptosis in vivo. To evaluate the mechanism of the enhanced antitumor effect of Ad-Endo combined with irradiation, the tumor sections were stained with the anti-CD31 antibody. As shown in Fig. 4A, tumors of the control groups (including NS group, Ad-null group, RT group, Ad-Endo group and Ad-null + RT group) exhibited larger microvessel counts than those of the Ad-Endo + RT group. The MVD in the Ad-Endo + RT group was $12.6 \pm 3.2 \%$, while the MVD was $49.4 \pm 8.2 \%$ in the Ad-Endo group, $74.3 \pm 4.6 \%$ in the Ad-null + RT group, $76.5 \pm 6.1 \%$ in the RT group, $88.9 \pm 6.4 \%$ in the Ad-null group and $90.2 \pm 3.7 \%$ in the NS group, respectively (Fig. 4B). 
A

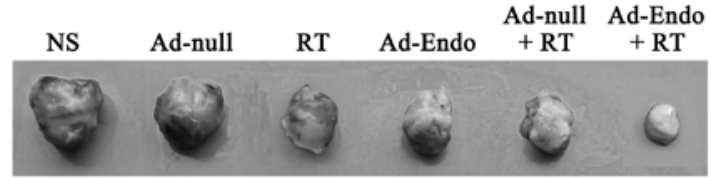

B

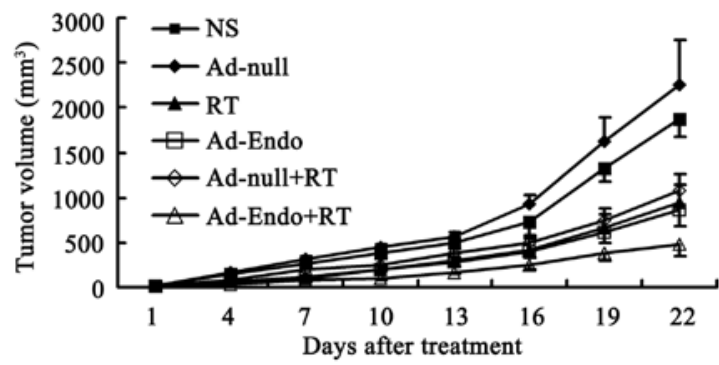

$\mathrm{C}$

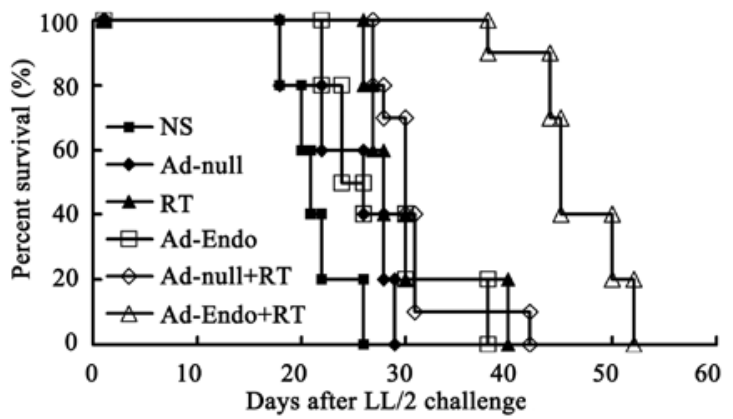

Figure 3. Effect of the combined Ad-Endo and radiotherapy treatment on the mouse LL/2 cell subcutaneous tumor model. (A) Tumor images of each group. (B) Changes in tumor volume of each group. (C) Life-span of the mice from each group.

\section{A}
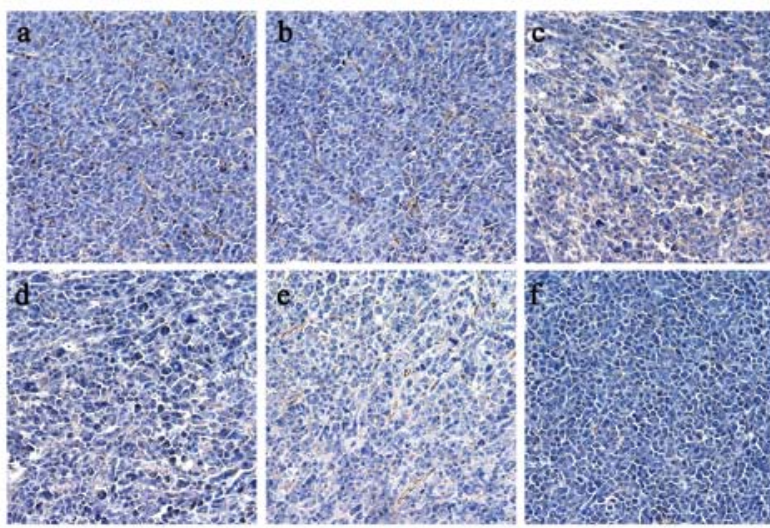

B

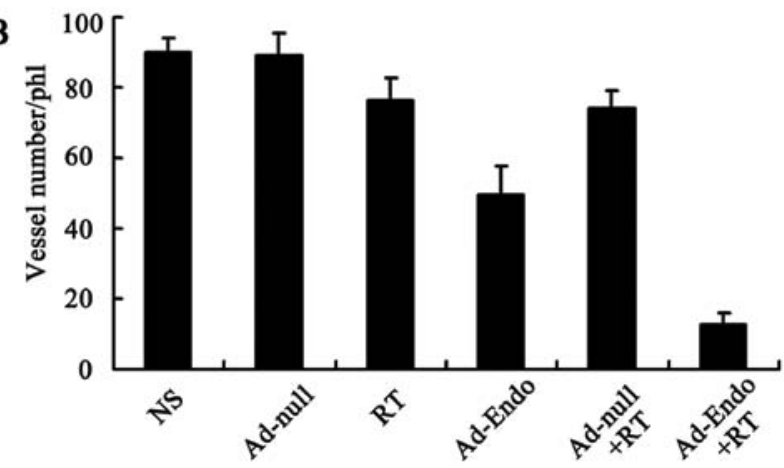

Figure 4. Immunhistochemical analysis of CD31 and microvessel density in the tumors. (A) Analysis of CD31 immunohistochemistry. (a) The NS group, (b) the Ad-null group, (c) the RT group, (d) the Ad-Endo group, (e) the Ad-null+RT group, (f) the Ad-Endo+RT group. (B) Analysis of microvessel density in the different treatment groups.
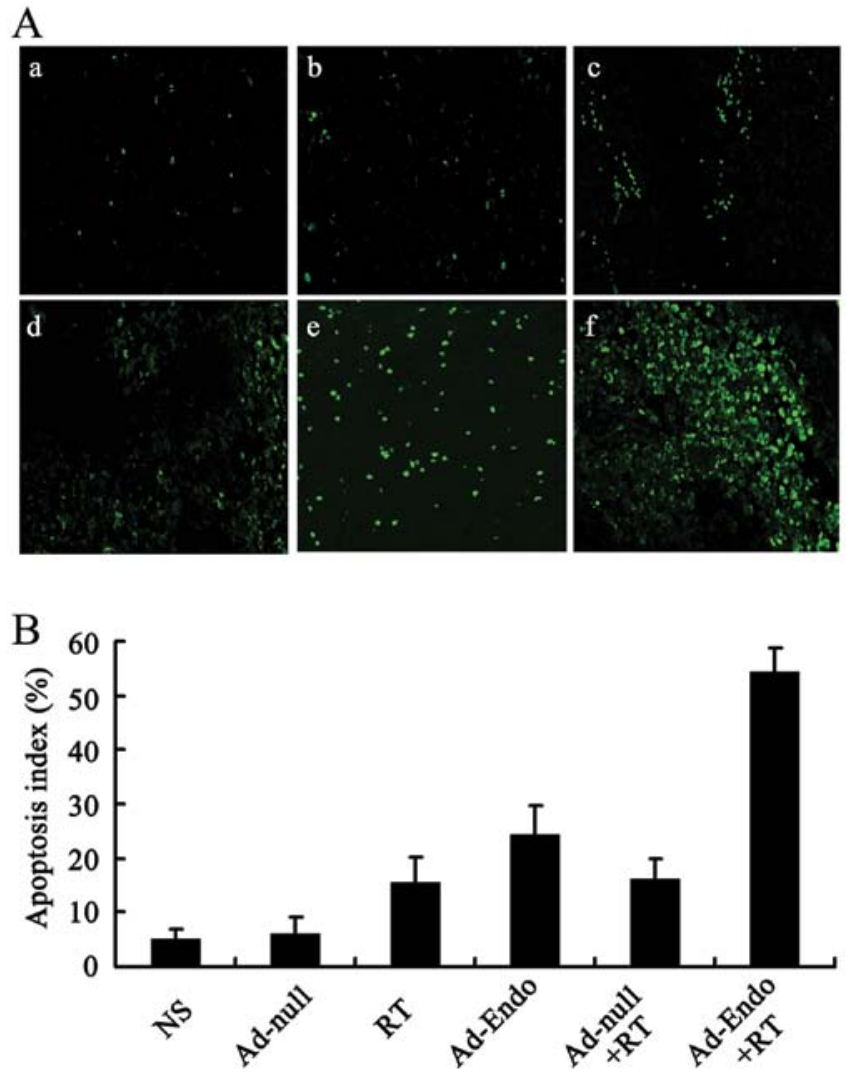

Figure 5. Analysis of apoptosis in the tumor cells by TUNEL. (A) TUNEL analysis in the tumor cells. (a) The NS group, (b) the Ad-null group, (c) the RT group, (d) the Ad-Endo group, (e) the Ad-null+RT group, (f) the Ad-Endo+RT group. (B) Apoptosis index of each group.

In order to estimate the apoptosis in tumor tissues, the tumor sections were stained with TUNEL reagent, and the positive results were confirmed as described in Materials and methods. Compared with the control groups, the number of apoptotic cells was increased in the Ad-Endo group, RT group and Ad-null + RT group. Furthermore, more obvious apoptosis was noted in the tumor sections of the Ad-Endo + RT group (Fig. 5A). In regards to the apoptotic index, the Ad-Endo + RT group showed the highest index among all of the groups (Fig. 5B). This suggests that Ad-Endo combined with irradiation obviously inhibited the angiogenesis in tumors, which promoted increased apoptosis.

Detection of anoxia factors with immunohistochemistry of $H I F-1 \alpha$. Tumor growth can induce anoxia in tissues, which increases the expression of HIF-1 $\alpha$. To assay the influence of Ad-Endo combined with irradiation on the level of anoxia factors, immunohistochemistry of HIF-1 $\alpha$ was applied. As shown in Fig. 6A and B, compared with the control groups, a decrease in expression of HIF-1 $\alpha$ was noted in the Ad-Endo, RT and Ad-null + RT groups. However, the combined Ad-Endo + RT group displayed the lowest level among all the groups, which was far less than those in the radiotherapy groups and the Ad-Endo group.

Inhibition of angiogenesis in the alginate encapsulation assay. Alginates can provide the desired marix for tumor angiogenesis 
A
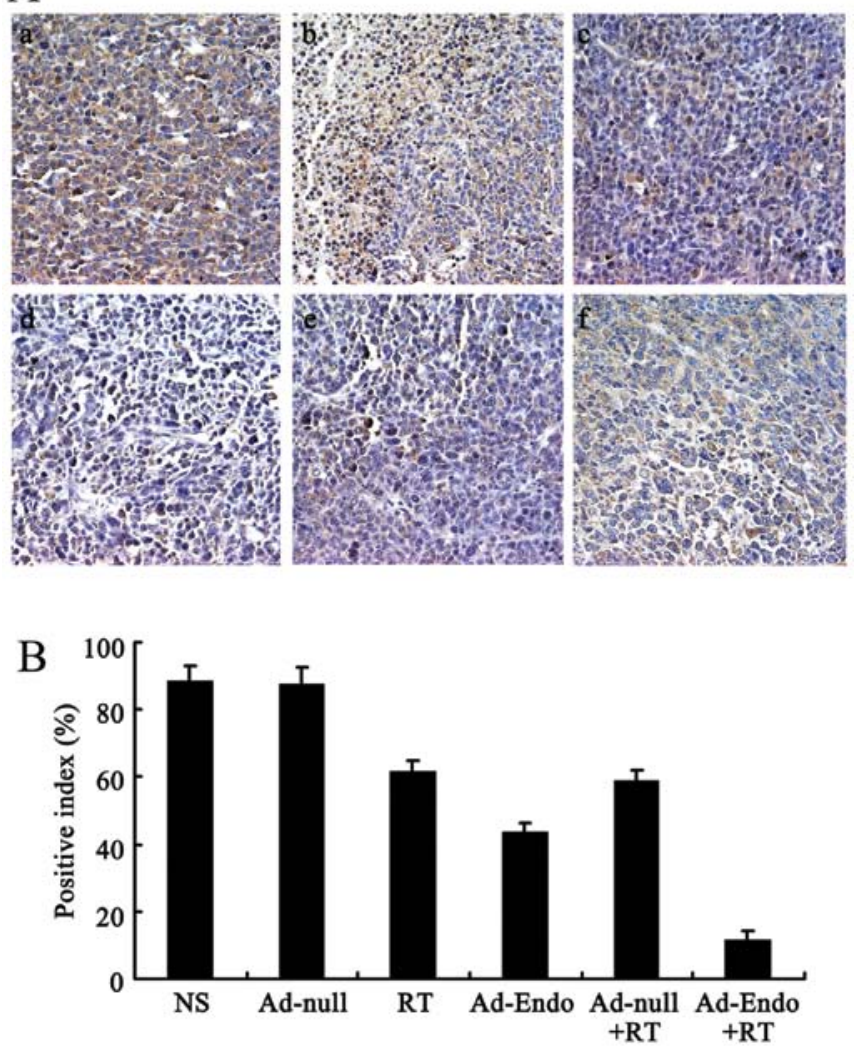

Figure 6. Immunohistochemical analysis of HIF-1 $\alpha$ in the tumors. (A) HIF-1 $\alpha$ immunohistochemistry. (a) The NS group, (b) the Ad-null group, (c) the RT group, (d) the Ad-Endo group, (e) the Ad-null+RT group, (f) the Ad-Endo+RT group. (B) Expression of HIF-1 $\alpha$.

and growth without affecting the release and delivery of cytokines and drugs. Thus, we evaluated the effect of Ad-Endo combined with irradiation on angiogenesis in vivo using the alginate encapsulation assay. As shown in Fig. 7A, there was less micrangium on the surface of beads in the Ad-Endo + RT group than that in the other groups. The FITC-dextran uptake was nearly $83 \%$ less in the Ad-Endo + RT group than that in the NS group or Ad-null group and $67-76 \%$ less than that in the other groups (Fig. 7B).

Combination treatment significantly inhibits tumor metastasis in the LL/2 cell pulmonary metastasis tumor model. Angiogenesis is not only relevant to tumor growth, but also promotes tumor metastasis. Thus, the LL/ 2 cell pulmonary metastasis tumor model was established and treatment with Ad-Endo combined with low-dose of irradiation was carried out. The lung weight of all mice is presented in Fig. 8A. The lung weight was $0.91 \pm 0.36 \mathrm{~g}$ in the NS group and $0.82 \pm 0.43 \mathrm{~g}$ in the Ad-null group; these weights were heavier than those in the RT group $(0.47 \pm 0.17 \mathrm{~g})$, Ad-Endo group $(0.54 \pm 0.22 \mathrm{~g})$ and Ad-null + RT group $(0.42 \pm 0.21 \mathrm{~g})$. Compared with the other groups, the Ad-Endo + RT group had the lowest lung weight $(0.2 \pm 0.06 \mathrm{~g})$. Moreover, the number of pulmonary metastatic nodules was counted, and the results are shown in Fig. 8B and Table I. The number of nodules in the NS and Ad-null groups was 180 and 165, respectively, for which metastatic nodules $>3 \mathrm{~mm}$ occupied 30 and $28 \%$. A smaller number of nodules
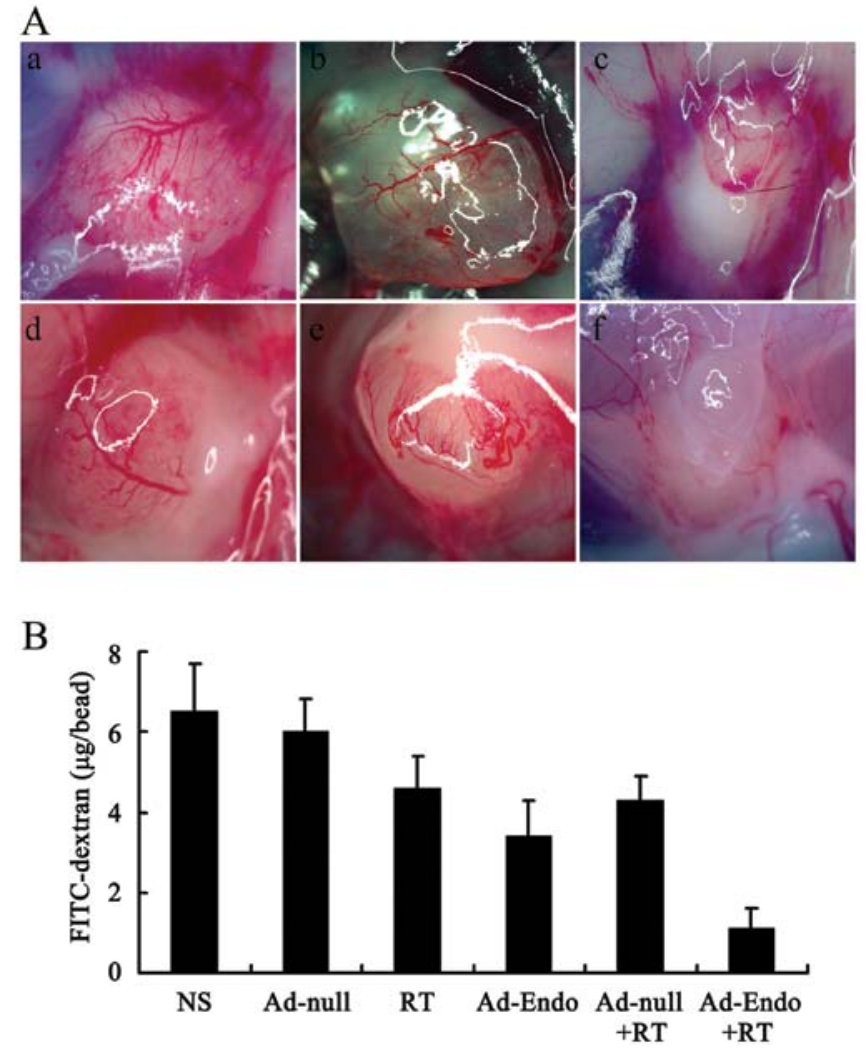

Figure 7. Observation of tumor angiogenesis by alginate implants. (A) Alginate beads as observed using microscopy. (a) The NS group, (b) the Ad-null group, (c) the RT group, (d) the Ad-Endo group, (e) the Ad-null+RT group and (f) the Ad-Endo+RT group. (B) The FITC-dextran uptake of alginate beads of each group.

was noted in the RT group (92), Ad-Endo group (110) and Ad-null + RT group (86), in which nodules $>3 \mathrm{~mm}$ accounted for 15,20 and $18 \%$, respectively. Notably, the number of nodules in the Ad-Endo + RT group was 10 and only $1.3 \%$ of nodules were $>3 \mathrm{~mm}$, which indicates that Ad-Endo combined with low-dose irradiation effectively inhibited the pulmonary metastasis of LL/2 cell tumors.

Observation of toxicity. In order to evaluate the potential sideeffects of the treatment, the toxicity assessment was executed in both models. There were no obvious differences in the gross measures among all the groups (data not shown). Moreover, no serious pathological changes in main organs were noted in the subcutaneous tumor model (Fig. 9) and pulmonary metastasis tumor model (data not shown).

\section{Discussion}

Angiogenesis plays an important role in tumor growth and metastasis (10,29,30). In 1945, Algire et al (31) proposed that 'the growth of tumor transplants is dependent on the development of a vascular system'. Subsequently, Folkman et al (32) found that tumor tissue could not exceed a size of 2-3 mm without the recruitment of new blood vessels, which supply nutrients and gases to tumors. Therefore, the authors hypothesized that the development of a vascular system was critical to the growth of tumors and suggested that antiangiogenesis may be an effective strategy for cancer therapy 
Table I. The number of pulmonary metastatic nodules in the LL/2 cell pulmonary metastasis tumor model.

\begin{tabular}{lcc}
\hline Group & $\begin{array}{c}\text { Median no. of } \\
\text { metastatic nodules }\end{array}$ & $\begin{array}{c}\% \text { large }(>3 \mathrm{~mm}) \\
\text { metastatic nodules }\end{array}$ \\
\hline NS & 180 & 30 \\
Ad-null & 165 & 28 \\
RT & 92 & 15 \\
Ad-Endo & 110 & 20 \\
Ad-null+RT & 86 & 18 \\
Ad-Endo+RT & 10 & 1.3 \\
\hline
\end{tabular}

Angiogenesis is a complex process which includes the proliferation and migration of vascular endothelial cells, formation of new capillaries and extracelluar matrix turnover (33-35). Several studies have shown that a host of cytokines regulates this process $(33,36,37)$. Although the mechanism is not clearly elucidated, endostatin is a significant factor which can inhibit endothelial cell proliferation and suppress the growth and metastasis of tumors $(18,38)$. Wickstrom et al (39) found that endostatin binds to $\alpha 5 \beta 1$ integrin and caveolin-1, which blocks the adhesion of endothelium and inhibits the activity of metalloproteases, further suppressing angiogenesis. Furthermore, endostatin can reduce the expression of Bcl-2 and Bel-XI, suppress the growth of endothelium and promote apoptosis, consequently inhibiting the growth and metastasis of tumors (15). Thus, endostatin is an effective target for tumor therapy. In the present study, we also demonstrated that endostatin can effectively inhibit the growth and metastasis of LL/2 tumors, and prolong the survival of tumorbearing mice.

Although research has shown that the recombinant protein of endostatin can effectively inhibit tumor growth and
A

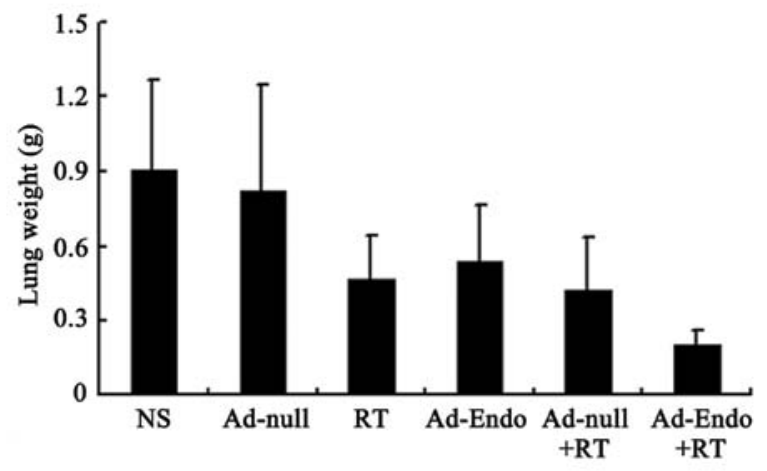

B

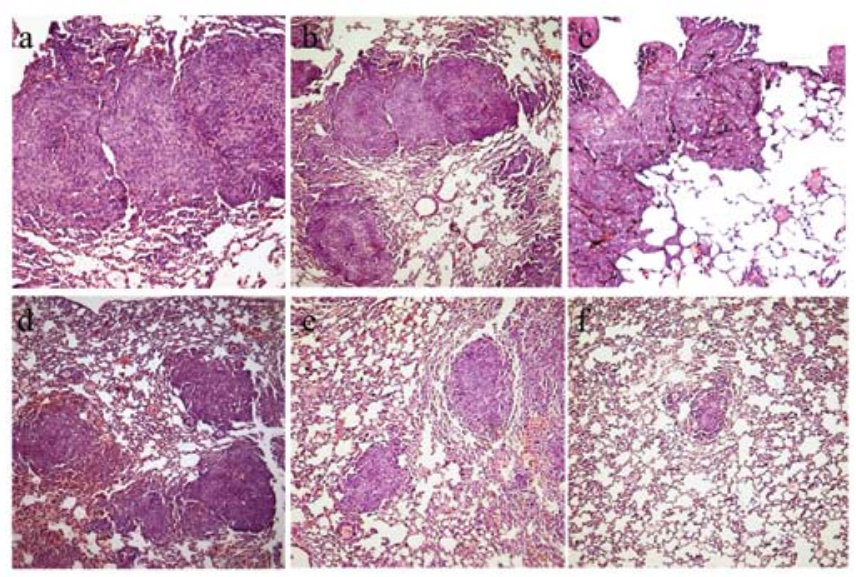

Figure 8. Antitumor effects of Ad-endo combined with irradiation in the LL2 cell lung metastasis tumor model. (A) Lung weight. (B) Analysis of lung tissue by H\&E. (a) The NS group, (b) the Ad-null group, (c) the RT group, (d) the Ad-Endo group, (e) the Ad-null+RT group, (f) the Ad-Endo+RT group. (B) The FITC-dextran uptake of alginate beads for each group.

metastasis, its widespread application has been hampered by difficulties in the large scale production of the protein and the high concentration of endostatin needed to maintain an anti-

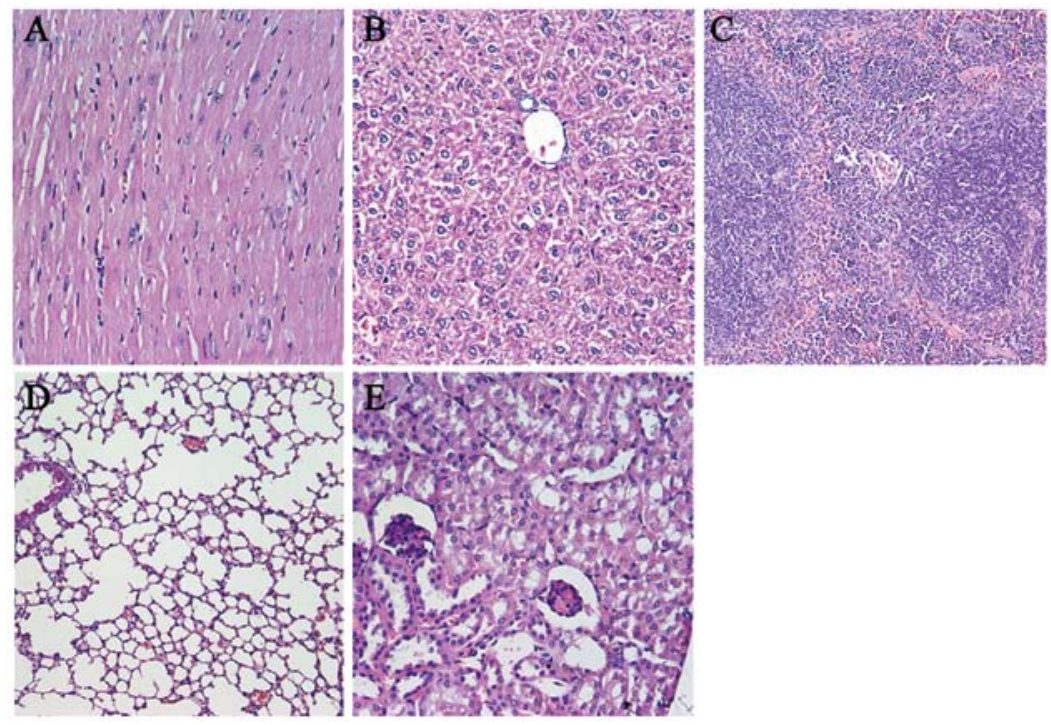

Figure 9. Observation of toxicity in the LL/2 cell subcutaneous tumor model. When the experiment was terminated, the mice in the Ad-Endo + RT group were sacrificed to obtain the main organs, and then paraffin tissue sections were constructed. H\&E analysis was subsequently perfomred. (A) Heart, (B) liver, (C) spleen, (D) lung and (E) kidney tissues. 
tumor effect $(36,40,41)$. Gene therapy with adenovirus vectors, which are gaining interest, have been reported to have beneficial effects in tumor treatment $(24,42-44)$. In the present study, an adenovirus expressing the secreted form of endostatin was identified as an effective strategy for endostatin gene therapy. After infection with Ad-GFP, LL/2 cells secreted GFP in a concentration-dependent manner. In addition, the expression of endostatin in vitro was examined by western blotting. Endostatin protein could be probed in the supernatant of LL/2 cells infected with Ad-Endo, which indicated that the adenovirus effectively transferred the endostatin gene to the LL/2 cells and that the endostatin gene was successfully expressed. Moreover, LL/2 cells infected with Ad-Endo stably and persistently expressed secretory endostatin which was confirmed by ELISA assay in our study.

However, as several angiogenesis factors are produced by endothelial cells and endostatin inhibits one of these factors, the inhibitory effect of endostatin is limited (3). Therefore, some researchers have combined conventional treatment strategies with endostatin to improve the effficacy of endostatin $(17,36,45,46)$. In the present study, we combined low-dose irradiation with Ad-Endo to treat the LL/2 cell subcutaneous model and pulmonary metastasis tumor model. Our study showed that either Ad-Endo or low-dose irradiation alone partially suppressed tumor growth and metastasis and prolonged the life-span of tumor-bearing mice along with no serious toxicity. Notably, the combination therapy showed a more effective antitumor activity when compared to the other groups. Although the mechanism of the enhanced antitumor efficacy remains to be elucidated, it may be related to a decrease in microvascular density and an increase in apoptosis in tumors. Our present findings revealed that there were less veins and more apoptotic cells in the tumors treated with Ad-Endo combined with irradiation when compared with the other groups. Although radiotherapy is an effective strategy to treat tumors through direct killing of cells, the activity is limited when hypoxia occurs in tumors (47-49). It is known that the rapid growth of tumors can cause regions of hypoxia in tumors, which contributes to the production of angiogenesis factors and blood vessel formation (48). Inhibition of angiogenesis with anti-angiogenic therapy may induce an increase in hypoxia in tumors, leading to radiotherapy resistance (49). However, other studies have shown that angiogenesis inhibitors can improve tumor oxygenation and increase the response to radiotherapy by delaying tumor growth (17,36,50-52). In the present study, when the tumors were treated with Ad-Endo, the blood vessel formation was inhibited and the vascular density was decreased. Consequently, hypoxia was improved, which boosted the activity of radiotherapy. Subsequently, more tumor cells were killed by irradiation, and tumor growth and metastasis were inhibited. These findings imply that radiotherapy and antiangiogenic therapy synergistically play a role in tumor treatment. Moreover, when patients are treated with effective doses of radiation, side-effects are commonly noted. In the present study, low-dose irradiation was adopted combined with Ad-Endo, which not only ensured an antitumor effect, but also inhibited the incidence of adverse side-effects.

In summary, our findings demonstrated that Ad-Endo combined with low-dose irradiation effectively inhibited the growth and metastasis of LL/2 cell tumors in a synergistic manner. The enhanced inhibitory effects were related to the decrease in microvascular density, inhibition of blood vessel formation, improvement in hypoxia and an increase in apoptosis of LL/2 cells. Further investigation of this strategy for the treatment of lung cancer and other tumors is warranted.

\section{Acknowledgements}

The study was supported by the National Natural Science Foundation of China (grant no. 81101604).

\section{References}

1. Jemal A, Siegel R, Ward E, et al: Cancer statistics, 2006. CA Cancer J Clin 56: 106-130, 2006.

2. Ning T, Yan X, Lu ZJ, et al: Gene therapy with the angiogenesis inhibitor endostatin in an orthotopic lung cancer murine model. Hum Gene Ther 20: 103-111, 2009.

3. Zhuang HQ and Yuan ZY: Process in the mechanisms of endostatin combined with radiotherapy. Cancer Lett 282: 9-13, 2009.

4. Govindan R, Bogart J and Vokes EE: Locally advanced non-small cell lung cancer: the past, present, and future. J Thorac Oncol 3: 917-928, 2008.

5. Bunn PA Jr and Thatcher N: Systemic treatment for advanced (stage IIIb/IV) non-small cell lung cancer: more treatment options; more things to consider. Conclusion. Oncologist 13 (Suppl 1): 37-46, 2008.

6. Bayman NA, Blackhall F, Jain P, Lee L, Thatcher N and FaivreFinn C: Management of unresectable stage III non-small-cell lung cancer with combined-modality therapy: a review of the current literature and recommendations for treatment. Clin Lung Cancer 9: 92-101, 2008.

7. Zhang HL, Yuan C, Zhang DM, et al: A novel combined conjugate vaccine: enhanced immunogenicity of bFGF with CRM197 as a carrier protein. Mol Med Rep 4: 857-863, 2011.

8. Carmeliet P and Jain RK: Angiogenesis in cancer and other diseases. Nature 407: 249-257, 2000.

9. Folkman J: Angiogenesis in cancer, vascular, rheumatoid and other disease. Nat Med 1: 27-31, 1995.

10. Hahnfeldt P, Panigrahy D, Folkman J and Hlatky L: Tumor development under angiogenic signaling: a dynamical theory of tumor growth, treatment response, and postvascular dormancy. Cancer Res 59: 4770-4775, 1999.

11. Shi HS, Yang LP, Wei W, et al: Systemically administered liposome-encapsulated Ad-PEDF potentiates the anti-cancer effects in mouse lung metastasis melanoma. J Transl Med 11: 86, 2013.

12. O'Reilly MS, Boehm T, Shing Y, et al: Endostatin: an endogenous inhibitor of angiogenesis and tumor growth. Cell 88: 277-285, 1997.

13. Oh SP, Warman ML, Seldin MF, et al: Cloning of cDNA and genomic DNA encoding human type XVIII collagen and localization of the $\alpha 1$ (XVIII) collagen gene to mouse chromosome 10 and human chromosome 21. Genomics 19: 494-499, 1994.

14. Wenzel D, Schmidt A, Reimann K, et al: Endostatin, the proteolytic fragment of collagen XVIII, induces vasorelaxation. Circ Res 98: 1203-1211, 2006.

15. Dhanabal M, Ramchandran R, Waterman MJ, et al: Endostatin induces endothelial cell apoptosis. J Biol Chem 274: 11721-11726, 1999.

16. Skovseth DK, Veuger MJ, Sorensen DR, De Angelis PM and Haraldsen G: Endostatin dramatically inhibits endothelial cell migration, vascular morphogenesis, and perivascular cell recruitment in vivo. Blood 105: 1044-1051, 2005.

17. Wu DS, Wu CM, Huang TH and Xie QD: Combined effects of radiotherapy and endostatin gene therapy in melanoma tumor model. Radiat Environ Biophys 47: 285-291, 2008.

18. Blezinger P, Wang J, Gondo M, et al: Systemic inhibition of tumor growth and tumor metastases by intramuscular administration of the endostatin gene. Nat Biotechnol 17: 343-348, 1999.

19. You ZY, Zhao Y, Liu F, Zhang YD and Wang JJ: The radiosensitization effects of Endostar on human lung squamous cancer cells H-520. Cancer Cell Int 10: 17, 2010. 
20. Cui R, Ohashi R, Takahashi F, et al: Signal transduction mediated by endostatin directly modulates cellular function of lung cancer cells in vitro. Cancer Sci 98: 830-837, 2007.

21. Thomas JP, Arzoomanian RZ, Alberti D, et al: Phase I pharmacokinetic and pharmacodynamic study of recombinant human endostatin in patients with advanced solid tumors. J Clin Oncol 21: 223-231, 2003

22. Li XP, Li CY, Li X, et al: Inhibition of human nasopharyngeal carcinoma growth and metastasis in mice by adenovirus-associated virus-mediated expression of human endostatin. Mol Cancer Ther 5: 1290-1298, 2006

23. Malecki M, Kolsut $P$ and Proczka R: Angiogenic and antiangiogenic gene therapy. Gene Ther 12 (Suppl 1): S159-S169, 2005.

24. Sauter BV, Martinet O, Zhang WJ, Mandeli J and Woo SL: Adenovirus-mediated gene transfer of endostatin in vivo results in high level of transgene expression and inhibition of tumor growth and metastases. Proc Natl Acad Sci USA 97: 4802-4807, 2000.

25. Mellon MJ, Ahn M, Jimenez JA, Kao C and Gardner TA: Antiangiogenic gene therapy for metastatic renal cell carcinoma produces tumor growth suppression in an athymic nude mouse model. J Urol 179: 737-742, 2008.

26. Kerbel RS, Yu J, Tran J, et al: Possible mechanisms of acquired resistance to anti-angiogenic drugs: implications for the use of combination therapy approaches. Cancer Metastasis Rev 20: 79-86, 2001.

27. Weidner N, Semple JP, Welch WR and Folkman J: Tumor angiogenesis and metastasis - correlation in invasive breast carcinoma. N Engl J Med 324: 1-8, 1991.

28. Wu Y, Yang L, Hu B, et al: Synergistic anti-tumor effect of recombinant human endostatin adenovirus combined with gemcitabine. Anticancer Drugs 16: 551-557, 2005.

29. Folkman J: Tumor angiogenesis and tissue factor. Nat Med 2 : 167-168, 1996

30. Bergers G, Javaherian K, Lo KM, Folkman J and Hanahan D: Effects of angiogenesis inhibitors on multistage carcinogenesis in mice. Science 284: 808-812, 1999.

31. Algire GH, Chalkley HW, Earle WE, et al: Vascular reactions of normal and malignant tissues in vivo. III. Vascular reactions of mice to fibroblasts treated in vitro with methylcholanthrene. J Natl Cancer Inst 11: 555-580, 1950.

32. Folkman J, Merler E, Abernathy $\mathrm{C}$ and Williams G: Isolation of a tumor factor responsible for angiogenesis. J Exp Med 133: $275-288,1971$

33. Risau W: Mechanisms of angiogenesis. Nature 386: 671-674, 1997.

34. Carmeliet P: Mechanisms of angiogenesis and arteriogenesis. Nat Med 6: 389-395, 2000

35. Folkman J and Kalluri R: Cancer without disease. Nature 427: $787,2004$.

36. Shi W, Teschendorf C, Muzyczka N and Siemann DW: Gene therapy delivery of endostatin enhances the treatment efficacy of radiation. Radiother Oncol 66: 1-9, 2003.

37. Ehrmann RL and Knoth M: Choriocarcinoma. Transfilter stimulation of vasoproliferation in the hamster cheek pouch Studied by light and electron microscopy. J Natl Cancer Inst 41: 1329-1341, 1968.
38. Huang $\mathrm{G}$ and Chen L: Recombinant human endostatin improves anti-tumor efficacy of paclitaxel by normalizing tumor vasculature in Lewis lung carcinoma. J Cancer Res Clin Oncol 136: 1201-1211, 2010.

39. Wickstrom SA, Alitalo K and Keski-Oja J: Endostatin associates with integrin $\alpha_{5} \beta_{1}$ and caveolin-1, and activates Src via a tyrosyl phosphatase-dependent pathway in human endothelial cells. Cancer Res 62: 5580-5589, 2002.

40. Lan KL, Ou-Yang F, Yen SH, Shih HL and Lan KH: Cationic liposome coupled endostatin gene for treatment of peritoneal colon cancer. Clin Exp Metastasis 27: 307-318, 2010

41. Boehm T, Pirie-Shepherd S, Trinh LB, Shiloach J and Folkman J: Disruption of the KEX1 gene in Pichia pastoris allows expression of full-length murine and human endostatin. Yeast 15: 563-572, 1999.

42. Griscelli F, Li H, Bennaceur-Griscelli A, et al: Angiostatin gene transfer: inhibition of tumor growth in vivo by blockage of endothelial cell proliferation associated with a mitosis arrest. Proc Natl Acad Sci USA 95: 6367-6372, 1998.

43. Lin P, Buxton JA, Acheson A, et al: Antiangiogenic gene therapy targeting the endothelium-specific receptor tyrosine kinase Tie2. Proc Natl Acad Sci USA 95: 8829-8834, 1998.

44. Regulier E, Paul S, Marigliano M, et al: Adenovirus-mediated delivery of antiangiogenic genes as an antitumor approach. Cancer Gene Ther 8: 45-54, 2001.

45. Bai RZ, Wu Y, Liu Q, et al: Suppression of lung cancer in murine model: treated by combination of recombinant human endostsatin adenovirus with low-dose cisplatin. J Exp Clin Cancer Res 28: 31, 2009.

46. Liao Z, Huang C, Zhou F, et al: Radiation enhances suicide gene therapy in radioresistant laryngeal squamous cell carcinoma via activation of a tumor-specific promoter. Cancer Lett 283: 20-28, 2009.

47. Durand RE and LePard NE: Contribution of transient blood flow to tumour hypoxia in mice. Acta Oncol 34: 317-323, 1995.

48. Bussink J, Kaanders JH and van der Kogel AJ: Tumor hypoxia at the micro-regional level: clinical relevance and predictive value of exogenous and endogenous hypoxic cell markers. Radiother Oncol 67: 3-15, 2003.

49. Cousins $C$ and Sharp C: Medical interventional proceduresreducing the radiation risks. Clin Radiol 59: 468-473, 2004.

50. Lee CG, Heijn M, di Tomaso E, et al: Anti-vascular endothelial grow th factor treatment augments tumor radiation response under normoxic or hypoxic conditions. Cancer Res 60: 5565-5570, 2000.

51. Teicher BA, Holden SA, Ara G, et al: Potentiation of cytotoxic cancer therapies by TNP-470 alone and with other anti-angiogenic agents. Int J Cancer 57: 920-925, 1994.

52. Jain RK: Normalizing tumor vasculature with anti-angiogenic therapy: a new paradigm for combination therapy. Nat Med 7: 987-989, 2001. 